\title{
A methodology development for the study of near surface stress gradients
}

\author{
M.J. Marques a, A.M. Dias ${ }^{\text {a,* }}$, P. Gergaud ${ }^{\text {b }}$, J.L. Lebrun ${ }^{\mathrm{c}}$ \\ ${ }^{a}$ GTR, Department of Mechanical Engineering, Universidade de Coimbra, Pólo II, Pinhal de Marrocos, P-3030 Coimbra, Portugal \\ ${ }^{\mathrm{b}}$ MATOP, ESA CNRS 6088, Université Aix-Marseille III, Faculté des Science St. Jérôme, 13397 Marseille cedex 20, France \\ ${ }^{\mathrm{c}}$ LM3, ESA CNRS 8006, ENSAM, 151 Bd. de l'Hôpital, F-75013 Paris, France
}

Received 17 June 1999; received in revised form 31 January 2000

\begin{abstract}
A modification of the geometry used in the $\sin ^{2} \psi$ technique of X-ray diffraction is described. A modified equation for residual stress determination, including geometric adapted $F_{\mathrm{ij}}$, is presented. This method allows near surface stress gradients determination and is called pseudo-grazing incidence method. The limits of the new technique were first tested on different powder materials with $\mathrm{X}$-ray radiation produced by conventional tubes and by a synchrotron radiation source. The technique was finally applied for the determination of a residual stress profile in a polished molybdenum surface before and after the deposition of a PVD chromium film. (C) 2000 Elsevier Science S.A. All rights reserved.
\end{abstract}

Keywords: Grazing incidence X-ray diffraction; Residual stress gradients; PVD chromium film

\section{Introduction}

Nowadays, residual stress evaluation by X-ray diffraction is a well known technique, and is frequently used for material characterisation. A detailed description of the state of the art can be found in the specialised literature [1-3]. When strong residual stress gradients are present within the depths range penetrated by X-ray, the normal procedure based in the conventional ' $\sin ^{2} \psi$ law' used to determine the residual stress must be changed. The penetration of X-rays can reach several hundred microns which can also correspond to the depth thickness of thin films or the depth range of surface treatments like grinding, machining, ion implantation, etc. If stress gradients are steep enough over the range of depth penetration of X-rays, the $\sin ^{2} \psi$ plots exhibit curvature. Such curvature can be used to estimate the variation of the stress components with depth [3]. However, this method has difficulties with the lack of sensitivity to stress gradients and the fact that several combinations of gradients may

\footnotetext{
* Corresponding author. Tel.: + 351-239-410653; fax: + 351-239829158.

E-mail address: gtr@gemini.ci.uc.pt (A.M. Dias)
}

cause the same curvature. In recent years, several authors [4-27] have studied the problem of the determination of residual stress gradients specially in the near surface region. Predecki et al. proposed in [4] two methods based on the grazing incident $\mathrm{X}$-ray diffraction for the asymmetric geometry in order to obtain the depth profiles of strains and stresses as a function of the penetration depth. Ruppersberg et al. proposed in [5] the universal-plot method, which can combine the $\sigma_{i i}(\tau)$-profiles obtained in $\Psi$-mode and $\Omega$-mode for different radiations and reflections in a 'master-curve' [6]. Their results indicated a strongly non-linear stress variation in a surface layer about $2 \mu \mathrm{m}$ thick. Genzel in [7] proposed the scattering-vector method which enables the evaluation of residual stress fields in materials which vary significantly within the penetration depth of the X-rays. His method has the great advantage to be applicable to textured materials [8,9]. Eigenman et al. $[10,11]$ had also worked on the problem of stress gradients and showed clearly that for very small penetration depths, the stress state may be considered as homogeneous in the sampled volume. Consequently, straight lines were found if $2 \theta_{\phi \psi}$ is plotted versus $\sin ^{2} \psi$. For increasing penetration depths the stress state in the volume sampled becomes more and more inhomoge- 
neous and curvature occurred to be more pronounced and shifted to higher $\psi$-values. All these efforts in order to analyse complicated non-uniform residual stress distributions $\sigma_{i j}(z)$ in the near-surface region of polycrystalline materials have been recently reviewed by Genzel in [12].

This study presents a modification of the conventional experimental geometry used in X-ray diffraction in order to evaluate the presence of residual stress gradients. This technique, called pseudo-grazing incidence X-ray diffraction (PGIXRD), can also be applied to the study of thin films, presenting the advantage to increase the path length of the radiation in the thin film which increases the film signal. Another advantage in the case of thin films is the elimination of the substrate signal which sometimes interferes with the film signal.

\section{Pseudo-grazing incidence method for residual stress evaluation}

\subsection{Theoretical background}

To determine the residual stress state in the sample by X-ray diffraction, the specimen must be rotated about two axes. One rotation is characterised by the tilt angle $\psi$ between the normal to the specimens surface $P_{3}$ and the normal $L_{3}$ to the diffracting planes of spacing $d_{h k l}$, which is parallel to the diffraction vector $\boldsymbol{Q}$. This

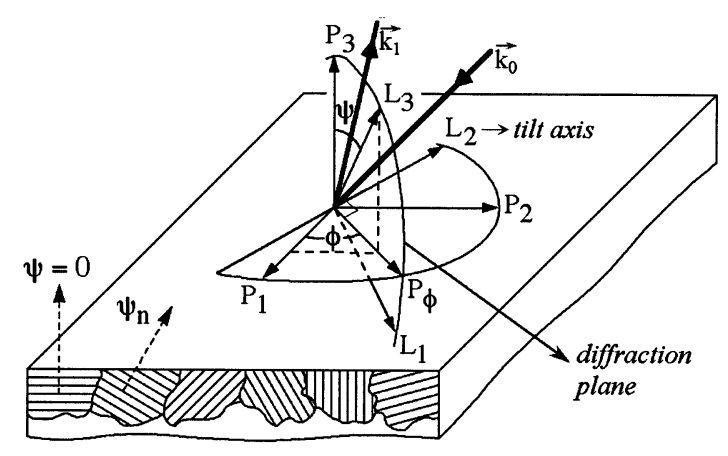

Fig. 1. $\Omega$-diffractometer.

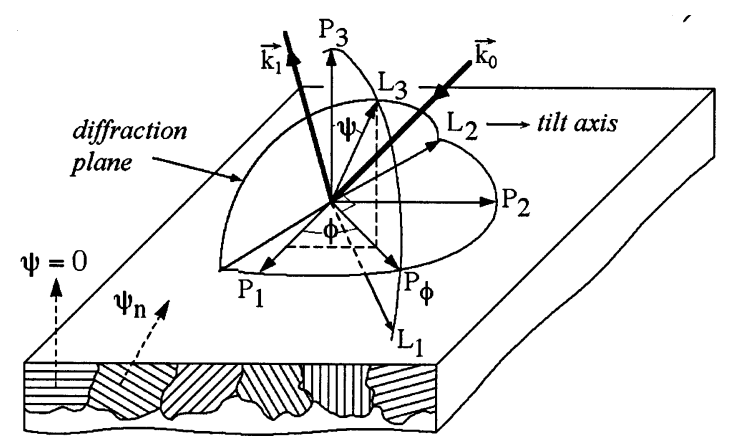

Fig. 2. $\Psi$-diffractometer. tilt can be accomplished by a rotation about an axis $L_{2}$ lying on the specimen surface and perpendicular to the diffraction plane, defined by the incident and the diffracted beams $k_{0}$ and $k_{1}$, respectively, i.e. using the $\Omega$-diffractometer (Fig. 1). It can also be achieved by a rotation about an axis lying on the specimen surface and belonging to the diffraction plane, but perpendicular to the diffraction vector $Q$, i.e. using the $\Psi$-diffractometer (Fig. 2). The second rotation is characterised by the azimuth angle $\phi$ and is executed about an axis parallel to the specimen normal $P_{3}$.

The residual or applied strain $\varepsilon_{\phi \psi}(h k l, \tau)$ can be determined from a change in the interplanar spacing $d_{\phi \psi}$ of the diffracting planes $(h k l)$, i.e.:

$\varepsilon_{\phi \psi}(h k l, \tau)=\frac{d_{\phi \psi}(h k l)-d_{0}}{d_{0}} \approx \ln \left(\frac{\sin \theta_{0}}{\sin \theta}\right)$

where $d_{0}$ is the interplanar spacing of the unstrained material and $\theta_{0}$ is the corresponding Bragg angle. $\tau$ is the penetration depth of X-rays which is defined by the condition that the intensity $I$ of the X-rays passing through the material is $1 / e$ of the primary intensity $I_{0}$.

The strains calculated from XRD data acquired at crystallographic scale $\varepsilon_{\phi \psi}(h k l, \tau)$, can be related to the components of the macrostress tensor $\sigma_{i j}(\tau)$ in the sample volume by:

$\varepsilon_{\phi \psi}=F_{i j} \sigma_{i j}(\tau)$

where the $F_{i j}$ are the generalised X-ray elasticity coefficients. When the material is macroscopically isotropic, the $F_{i j}$ coefficients vary linearly with $\sin ^{2} \psi$. Using X-ray elastic constants (XEC) $\frac{1}{2} S_{2}(h k l)$ and $S_{1}(h k l)$ and diffraction vector angles $\phi$ and $\psi$, the $F_{i j}$ are defined as follows:

$F_{11}=\frac{1}{2} S_{2}(h k l) \cos ^{2} \phi \sin ^{2} \psi+S_{1}(h k l)$
$F_{12}=\frac{1}{2} S_{2}(h k l) \sin 2 \phi \sin ^{2} \psi$
$F_{22}=\frac{1}{2} S_{2}(h k l) \sin ^{2} \phi \sin ^{2} \psi+S_{1}(h k l)$

$F_{13}=\frac{1}{2} S_{2}(h k l) \cos 2 \phi \sin 2 \psi$

$F_{33}=\frac{1}{2} S_{2}(h k l) \cos ^{2} \phi+S_{1}(h k l)$

$F_{23}=\frac{1}{2} S_{2}(h k l) \sin \phi \sin 2 \psi$

Assuming an exponential law, the strains $\varepsilon_{\phi \psi}(h k l, \tau)$, obtained by measuring the interplanar spacing $d_{\phi \psi}(h k l)$ at various tilt $\phi$ and $\psi$ of the sample with respect to $\mathrm{X}$-ray beam (Eq. (1)), are averages with respect to the depth $z$ below the surface: 


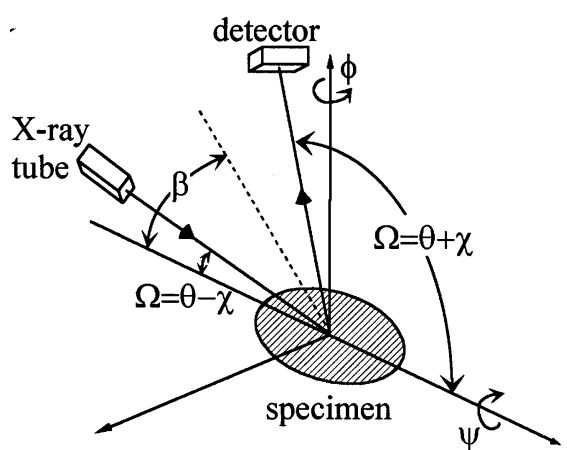

Fig. 3. Shreme of pseudo-grazing incidence geometry.

$\varepsilon_{\phi \psi}(h k l, \tau)=\frac{\int_{0} \varepsilon_{\phi \psi}(h k l, z) e^{-\frac{\tau}{\tau} d z}}{\int_{0}^{D} e^{-\frac{z}{\tau}} d z}$

$D$ is the thickness of the sample investigated. If $D$ is large compared to the penetration depth $\tau$, the upper limit of the integrals in Eq. (4) can be replaced by infinity and one finds:

$\varepsilon_{\phi \psi}(h k l, z)=\frac{\int_{0}^{\infty} \varepsilon_{\phi \psi}(h k l, z) e^{-\frac{z}{\tau}} d z}{\tau}$

With a similar reasoning for the components of the macrostress tensor $\sigma_{i j}(\tau)$, one finds:

$\sigma_{i j}(\tau)=\frac{\int_{0}^{\infty} \sigma_{i j}(z) e^{-\frac{z}{\tau}} d z}{\tau}$

From Eqs. (5) and (6), it can be observed that the average deformation and the stress profiles obtained by X-ray diffraction are the Laplace transform of the actual depth profiles $\varepsilon_{\phi \psi}(h k l, z)$ and $\sigma_{i j}(z)$ with respect to $1 / \tau$, divided by $\tau$. Thus, in order to obtain $\varepsilon_{\phi \psi}(h k l, z)$ or $\sigma_{i j}(z)$, the experimental $\varepsilon_{\phi \psi}(h k l, \tau)$ or $\sigma_{i j}(\tau)$ data are fitted by a well-behaved function such that a valid inverse Laplace transform exists for $\tau$ times this function $[4,13]$. Another procedure used to convert the $\tau$-profiles in $z$-profiles by means of Eq. (4) imposes analytical assumptions for the $\varepsilon_{i j}(z)$ profiles. Usually exponential or polynomial functions are used $[5,6,10,11]$. Recently, it has been developed a numerical procedure in order to solve Eq. (4) without restriction on $D[14]$.

\subsection{The pseudo-grazing incidence method}

The experimental set up in a $\Psi$-goniometer is always symmetrical relative to the sample's surface: the detector always moves at angle $2 \theta$, while the sample moves at $\theta, \theta$ being the Bragg angle. For this geometry the penetration depth $\tau$ can be calculated by the following equation $[15,16]$ : $\tau=\frac{\cos \psi \sin \theta}{2 \mu}$

where $\mu$ is the absorption coefficient of the irradiated material for the X-ray wavelength used. If this formula is applied, for instance, to an ferritic steel, irradiated with a chromium wavelength, the penetration depth is in the order of $6 \mu \mathrm{m}$, for $2 \theta=156^{\circ}$ and $\psi=0^{\circ}$.

A four-circle goniometer is required to implement the PGIXRD. A smaller penetration depth of the radiation is achieved by reducing the incident angle $\Omega$, defined as the angle between the incident beam and the sample surface. The penetration depth $\tau$ in this grazing incident geometry can be estimated by the equation $[15,16]$ :

$\tau=\frac{\cos \psi\left(\sin ^{2} \theta-\sin ^{2} \chi\right)}{2 \mu \sin \theta \cos \chi}$

where $\chi$ is the rotation of the sample, i.e. $\chi=\theta-\Omega$.

The idea underlying the method presented in this paper can be easily understood from Eq. (8). If the incident angle is reduced the penetration depth will also decrease and, by choosing different incident angles it will be possible to reach different surface layers of the material. The method however can only be applied if the residual stress gradient over the respective range of $\mathrm{X}$-ray penetration depth is not steep, i.e. the stress is assumed to be homogeneous in each of those layers. In the following, we will use a mean value $z^{*}$ which was defined as the penetration depth at half/mid $\psi$ maximum value (for $-60^{\circ}<\psi<60^{\circ}, z^{*}=\tau_{\psi=30^{\circ}}$ ). The geometry proposed is presented in Fig. 3. The $\Omega$ incident angle must remain constant while the sample is rotated in a plane perpendicular to the plane defined by the incident and the diffracted X-ray beam in order to determine the residual stress, as in the conventional $\Psi$-geometry. In other words, the sample must rotate in different $\psi$ inclinations. This new geometry is no longer a symmetric one, and $\Omega$ is no longer equal to the Bragg angle. It also means that for $\psi=0^{\circ}$ the diffracting planes are no more parallel to the sample surface. Using different $\Omega$ incident angles, the average penetration depth $z^{*}$ can be changed and the respective residual stresses are obtained as mean values over layers of different thickness.

The three angles $\Omega, \psi, \phi$, which represent this new geometry, must be considered, and, applying theory of elasticity, the generalised X-ray elastic coefficients $F_{i j}$ can be obtained by the following equation [17]:

$F_{11}=\frac{1}{2} S_{2}(h k l)\left(\cos ^{2} \phi \sin ^{2} \psi \sin ^{2} \beta+\sin ^{2} \phi \cos ^{2} \beta\right.$

$-1 / 2 \sin 2 \phi \sin \psi \sin 2 \beta)+S_{1}(h k l)$

$F_{22}=\frac{1}{2} S_{2}(h k l)\left(\cos ^{2} \phi \cos ^{2} \beta+\sin ^{2} \phi \sin ^{2} \psi \sin ^{2} \beta\right.$

$-1 / 2 \sin 2 \phi \sin \psi \sin 2 \beta)+S_{1}(h k l)$

$F_{33}=\frac{1}{2} S_{2}(h k l)\left(\cos ^{2} \psi \sin ^{2} \beta\right)+S_{1}(h k l)$ 
$F_{12}=\frac{1}{2} S_{2}(h k l)\left(\sin 2 \phi\left(\sin ^{2} \psi \sin ^{2} \beta-\sin ^{2} \beta\right)\right.$

$+\cos 2 \phi \sin \psi \sin 2 \beta)$

$F_{13}=\frac{1}{2} S_{2}(h k l)\left(\cos \phi \sin 2 \psi \sin ^{2} \beta-\cos \phi \sin \psi \sin 2 \beta\right)$

$F_{23}=\frac{1}{2} S_{2}(h k l)\left(\sin \phi \sin 2 \psi \sin ^{2} \beta+\cos \phi \cos \psi \sin 2 \beta\right)$

The angle $\beta$ in Eq. (9) describes the angle between the bisecting-line of incident and diffracted beam and the surface of the material, i.e. $\beta=\pi / 2-\chi \quad$ (or $\beta=\pi / 2-\theta+\Omega)$.

The $F_{i j}$ determined by means of the Eq. (9) suppose that $\frac{1}{2} S_{2}(h k l)$ and $S_{1}(h k l)$ are constant over the diffracting volume of the material. This condition is fulfilled if there is no crystallographic texture and if surface anisotropy can be neglected. Concerning the near surface analysis, additional effects could influence XRD results. Effectively, surface stress and surface strain, as defined by Nozières et al. [18] and following Gibbs surface definition [19], may occur. The validity of bulk elastic constants for describing thin film properties is therefore arising. A simple model of size dependence of the biaxial modulus of thin films had been published [20]. In this paper, Streitz et al. [20] had calculated the thickness-dependent biaxial modulus of thin metal films. It seems that a film of roughly few monolayers $(0.5-1 \mathrm{~nm})$ can already be considered as an elastic continuum where bulk constants are valid as confirmed by $[21,22]$. In our present study the lower mean penetration depth is in the order of $100 \mathrm{~nm}$. The surface effect can therefore be neglected, as others authors had done $[5,15,23]$. A general overview of problem in the case of near surface XRD analysis is mentioned by Ruppersberg et al. in [6] and by Genzel in [12].

\section{Calibration of the pseudo-grazing incidence method on powders; experimental limits}

\subsection{Experimental procedure}

The PGIXRD had been tested using powders of different materials, irradiated by both conventional

Table 1

Powder materials and X-ray wavelengths used to calibrate the PGIXRD technique

\begin{tabular}{llll}
\hline Powder & X-ray wavelength & $\{h k l\}$ & $2 \theta_{0}\left(^{\circ}\right)$ \\
\hline Copper & Synchrotron radiation, & $\{420\}$ & 127 \\
& $\lambda=0.1450 \mathrm{~nm}$ & & \\
Iron & $\mathrm{Cu} \mathrm{K} \alpha, \lambda=0.15418 \mathrm{~nm}$ & $\{310\}$ & 116.52 \\
Nickel & $\mathrm{Cu} \mathrm{K} \alpha, \lambda=0.15418 \mathrm{~nm}$ & $\{420\}$ & 156.09 \\
Molybdenum & $\mathrm{Cu} \mathrm{K} \alpha, \lambda=0.15418 \mathrm{~nm}$ & $\{321\}$ & 132.36 \\
\hline
\end{tabular}

X-ray tubes and synchrotron radiation, as listed in Table 1. The purpose was to determine what problems were introduced by the new asymmetric geometry itself. The powders were commercial, pure and presented small grain size $(2-5 \mu \mathrm{m})$. They were annealed to eliminate any residual stress. Thin layer of these powders had been deposited on a flat glass substrate. The peak position of the powders without stresses cannot change, no matter what geometry is used. The tests were made observing the powder peak position as a function of $\psi$.

The tests using conventional X-ray radiation were performed on a Seifert 4-circle PTS goniometer, equipped with a CCD germanium detector, which has high energy resolution. To minimise defocusing problems, a Soller slit with $0.4 \mathrm{rad}$ divergence was used in front of the detector [24]. A collimator with a 2 $\mathrm{mm}$ circular aperture was used in the incident X-ray beam. The peak displacement was analysed for $11 \psi$ between $\pm 60^{\circ}$, for different $\Omega$ incident angles between the $\theta$ angle and $2^{\circ}$. The peak acquisitions were made step by step, using a fixed step size equal to $0.1^{\circ}$. On the other hand, the step time was increased for the acquisitions corresponding to lower incident angles. For instance, step times equal to 20 and $100 \mathrm{~s}$ was used for the acquisitions with an incident angle equal to the Bragg angle and with an incident angle equal to $2^{\circ}$, respectively, for the iron powder acquisitions. The reproducibility of the results was verified by repeating each acquisition five times.

The synchrotron radiation, obtained by the acceleration of high energy particles, is a coherent beam of higher intensity than the X-ray beams obtained with conventional tubes. Several authors referred to the great advantages of the use of this kind of radiation for residual stress evaluation $[6,11,28]$. Because of the beam characteristics, the PGIXRD was also tested with this kind of radiation. The experiments were carried out on copper powder, using a wavelength of $0.145 \mathrm{~nm}$ for incident angles equal to $\theta, 20^{\circ}, 10^{\circ}, 5^{\circ}$ and $2^{\circ}$, in Laboratoire pour l'Utilisation du Rayonnement Électromagnétique (LURE), Centre Universitaire Paris-Sud, Orsay, France, at beam line D23. This beam line is equipped with two silicium monochromators. The first permits wavelength selection, and the second, which is able to move horizontally, reflects the beam to a fixed exit and focuses the beam in the horizontal plane. The experiments were carried out using a Huber 4-circle goniometer equipped with a scintillator. The detector has a germanium monochromator to prevent problems with fluorescence. 


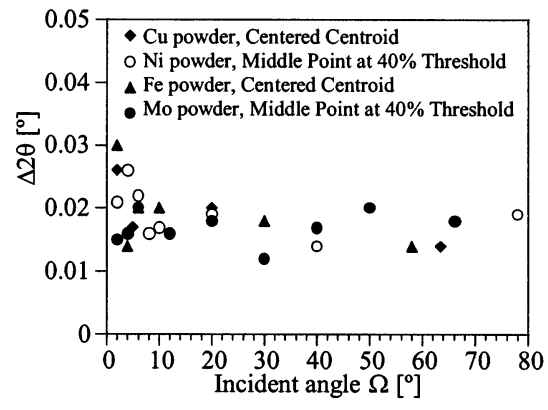

Fig. 4. Peak shifts vs. incident angle for different powders.

Several procedure may localise the X-ray diffracted peaks, and then different methods may be used to treat these peaks in order to determine the residual stress state. The choice of the correct method is important for the X-ray diffraction technique to be reliable. However, it should be noted that these methods can only be applied if the peak shape does not change $[1,2]$. Sometimes these changes are scarcely perceptible, and, depending on the software used, they can be disregarded. In this work, three methods were used to obtain improved results, especially at the lower incident angles. The methods employed are namely as the 'centroid', the 'centered centroid' and the 'middle point at $40 \%$ threshold' methods [1].

\subsection{Results and discussion}

Hauk recommends [29] for a good alignment of diffractometer in the complete range of $\psi$ that the peak positions of the $\{h k l\}$-lines of the calibration powder should not differ by more than $\pm 0.01^{\circ}$ in $2 \theta$ scale. However, in our laboratories an internal procedure is used which states for the determination of residual stress by X-ray diffraction that the peak displacement of a powder without stresses must not exceed $0.02^{\circ}$ in the $2 \theta$ scale.

The results shown in Fig. 4 are related to the grazing incidence geometry itself. The intensity of the peaks is very weak for the smaller $\Omega$ angles, and it worsens as the $\psi$ angle increases, because the volume of material able to diffract decreases. Fig. 5 gives two examples of peak intensity variation with the $\psi$ angle, for normal incidence and for an incident angle equal to $2^{\circ}$. In the normal incidence, i.e. $\Omega=\theta$, the peaks have the same order of intensity for $\psi=0^{\circ}$ and $\psi=60^{\circ}$. However, for smaller incident angles the peak intensity is very weak and decreases with increasing $\psi$ angle. Fig. 6 shows the relation of the mean peak intensity versus $\Omega$ incident angle for nickel powder diffraction. It can be seen that the peak intensity decreases strongly when the incident angle $\Omega$ becames smaller than $10^{\circ}$. Another reason for the peak shifts observed may be caused by sistematic deviations from the focusing Bragg-Brentano geometry

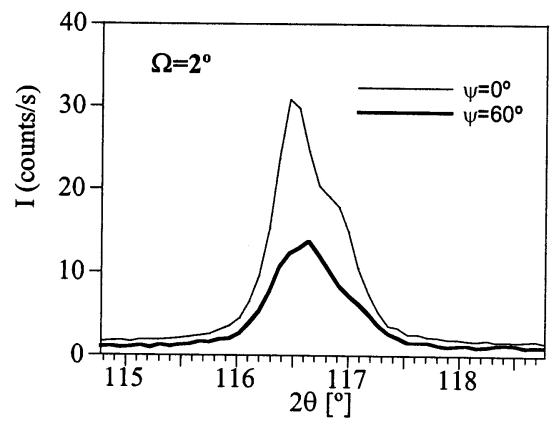

a) iron powder
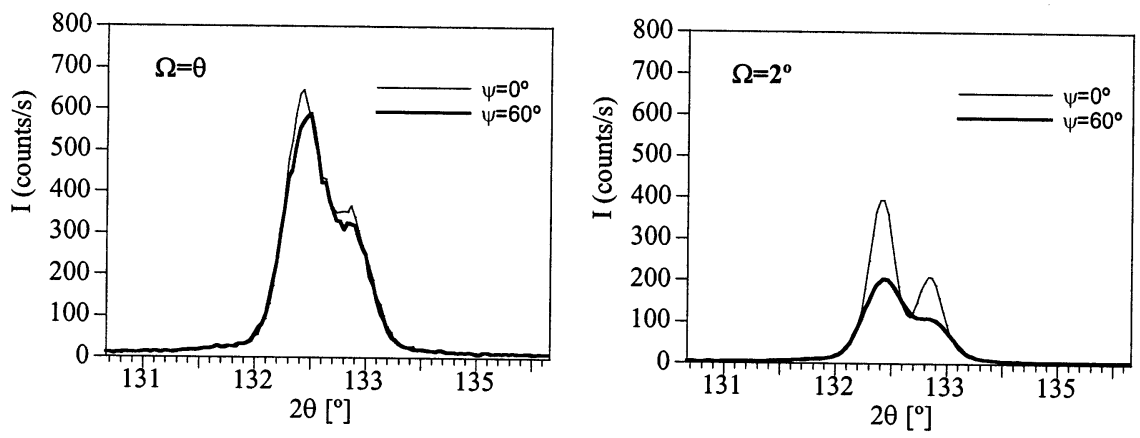

b) molybdenum powder

Fig. 5. Peak shape acquired with $\mathrm{Cu} \mathrm{K} \alpha$ radiation: (a) iron powder, (b) molybdenum powder. 


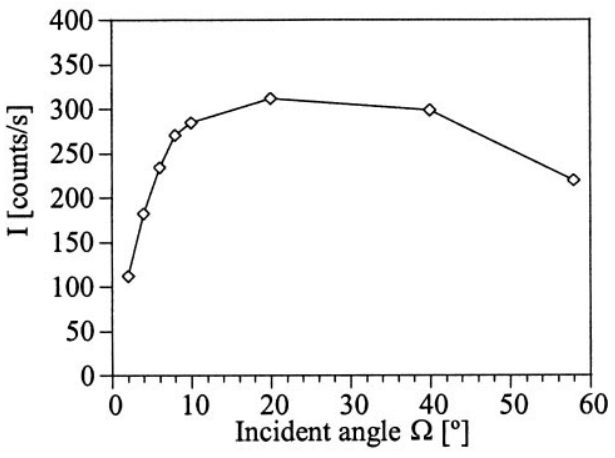

Fig. 6. Peak intensity of the nickel powder vs. $\Omega$ incident angle.

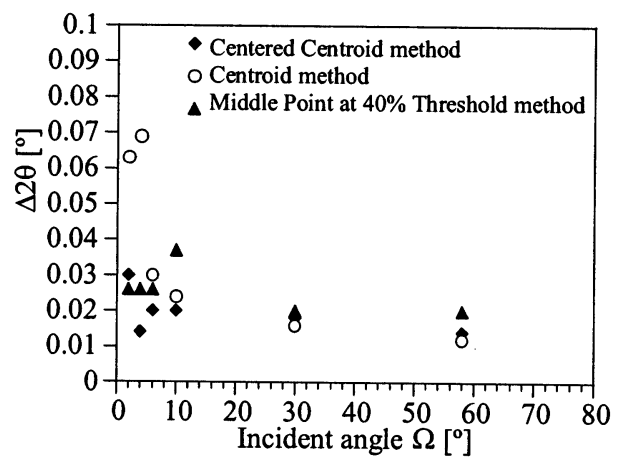

a) iron powder

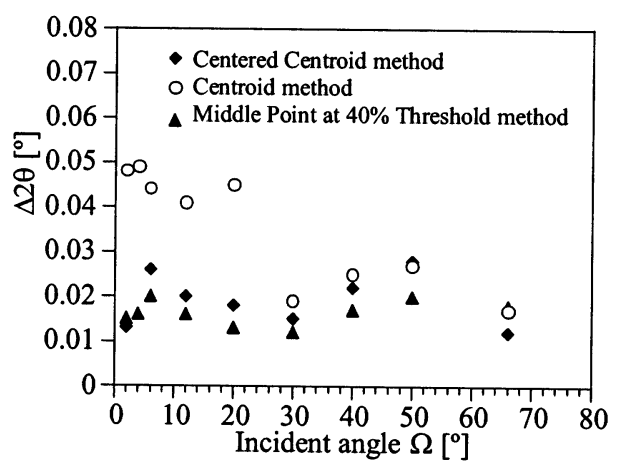

b) molybdenum powder

Fig. 7. Influence of the peak treatment method on the peak position: (a) iron powder; (b) molybdenum powder.

for flat samples and finite horizontal divergence. For the conventional $\Psi$-mode $\mathrm{X}$-ray acquisitions, the peak broadening is nearly symmetric over the range of $\psi$ used, and consequently the effect can be neglected. However, for $\Omega$-mode, broadening becames asymmetric and may lead to a sensible peak shift. As the experimental apparatus of pseudo-grazing incidence is a combination of $\Psi$-mode and $\Omega$-mode, it is not surprising the effect of peak shift observed for the lower incident angles. Similar effect had been also stated by Genzel [7].
We will now consider the influence of the method of peak position determination. With the centroid method, the variation of peak position versus incident angle $\Omega$ was higher than with the other methods, especially at lower incident angles, as can be seen in Fig. 7. It was observed that the change in the peak shape (see for instance, the change in the peak shape in a molybdenum powder at $\Omega=2^{\circ}$, in Fig. 5(b) plays a more important role in the centroid method than it does in the others. We do not recommend this method for the implementation of the PGIXRD technique.

The changes in the powders' peak shape at the lower incident angles were more pronounced for the cases in which a clear separation of $K \alpha_{1}$ and $K \alpha_{2}$ lines occurred. The variation in the peak position for each $\psi$ at lower incident angles was higher for the most asymmetric peak, i.e. for the nickel and molybdenum powders. It was further observed that this variation had a stronger effect when the centroid or the centered centroid method was applied. Fig. 5 gives an example of the peak shape of iron and molybdenum powders. As may be seen, the $K \alpha$ doublet of the molybdenum peak is more visible than that of the iron peak. This fact, together with the results shown in Fig. 7, led us to the conclusion that the middle point at $40 \%$ threshold method is the best method for treating results of this type. The centroid and the centered centroid methods are very sensitive to this kind of shape and should therefore be omitted. The ratio between the intensity of $K \alpha_{1} / K \alpha_{2}$ is not constant at the lower incident angles, probably because there is insufficient material that is able to diffract, and these two methods are very sensitive to such change. We also believe that the instabilities of the peak shape at lower $\Omega$ angles imply individual peak treatment for each $\psi$.

The question of peak shape should always be kept in mind in future applications of the PGIXRD technique, with a particular attention at lower incident angles.

The results obtained with synchrotron radiation are given in Fig. 4 (copper powder). It can be seen that the peak position versus $\Omega$ incident angle is similar to that given by the conventional X-ray beam. However, the D23 beam line has the disadvantage that the detector movements are limited to a $2 \theta$ maximum of $130^{\circ}$. This limitation imposed the choice of an angle of $127^{\circ}$ for the copper powder. From the restricted viewpoint of residual stress evaluation, the technique works much better with higher $2 \theta$ angles. Another problem is that of copper fluorescence, which could be avoided if a CCD detector were used.

To sum up, since the results obtained by PGIXRD may introduce 'ghost' residual stresses, especially at lower incident angles, the goniometer must be well aligned using, for instance, a powder without stress for data comparison. This has to be done very carefully, because peak displacement problems always arise at the lower incident angles. 
Table 2

Average penetration depth $z^{*}$ of the $\mathrm{Cu} \mathrm{K} \alpha$ for Mo (321) family planes, for different $\Omega$ incident angles

\begin{tabular}{lccccccc}
\hline Angle of incidence $(\Omega)$ & $66^{\circ}(\Omega=\theta)$ & $40^{\circ}$ & $20^{\circ}$ & $10^{\circ}$ & $8^{\circ}$ & $6^{\circ}$ & $4^{\circ}$ \\
\hline$z^{*}(\mathrm{~nm})\left(z^{*}=\tau_{\psi=30^{\circ}}\right)$ & 2390 & 2050 & 1310 & 750 & 620 & 480 & 340 \\
\hline
\end{tabular}

Table 3

Average penetration depth $z^{*}$ of the $\mathrm{Cr} \mathrm{K} \alpha$ for Mo (211) family planes, for different $\Omega$ incident angles

\begin{tabular}{lccccccc}
\hline Angle of incidence $(\Omega)$ & $66^{\circ}(\Omega=\theta)$ & $40^{\circ}$ & $20^{\circ}$ & $10^{\circ}$ & $8^{\circ}$ & $6^{\circ}$ & $4^{\circ}$ \\
\hline$z^{*}(\mathrm{~nm})\left(z^{*}=\tau_{\psi=30^{\circ}}\right)$ & 820 & 720 & 460 & 270 & 250 & 200 & 140 \\
\hline
\end{tabular}

4. An application of the pseudo-grazing incidence technique: characterisation of a polished molybdenum surface before and after chromium deposition

A molybdenum surface was polished mechanically before the physical vapour deposition (PVD) of a chromium coating. The residual stress state of this surface was analysed before and after the chromium deposition. The aim was to determine whether a residual stress gradient was present in the surface and to study the influence of the deposition of the film on that initial residual stress state.

The Mo substrate and the Cr coating were $1.5 \mathrm{~mm}$ and $1500 \mathrm{~nm}$ thick, respectively. The $\mathrm{Cr}$ thin film have been deposited by DC magnetron sputtering using a chrome planar target and a pure argon as a sputtering gas. The parameters of the deposition was: pressure $0.2 \mathrm{~Pa}$, substrate temperature $250^{\circ} \mathrm{C}$, without substrate bias.

Three pole figures of the Mo substrate enables us to check the texture. It was observed a very weak fibre texture $<200>$. The small variation of intensity in pole figures allows us to neglect the existence of the texture in the Mo substrate which will be treated as a quasi-isotropic material.

The residual stresses in the Mo sample were determined by PGIXRD with $\mathrm{Cu} \mathrm{K} \alpha$ and $\mathrm{Cr} \mathrm{K} \alpha$ radiation at several $\Omega$ incident angles, which made different average penetration depths $z^{*}$ possible, as shown in Table 2 and Table 3. After coating deposition, the residual stress state of the Mo substrate was again examined, using the same procedure.

The residual stresses in the $\mathrm{Cr}$ coating were also determined. However, the procedure for evaluating the residual stress in the film is different from the procedure described in this work. The film is strongly textured, as often observed with PVD films, and this implies a different approach for evaluating residual stress by means of X-ray diffraction. The method used is usually referred as the crystallite group method [30-32], or sometimes, as the ideal directions method. In this procedure, several family planes $\{h k l\}$ are needed to determine the stress. First, the texture of the film was examined by the three pole figures, $\{110\},\{200\}$ and $\{211\}$, with $\mathrm{Cu} \mathrm{K} \alpha$, permitting the identification of a fibre texture $<100>$. The $\{h k l\}$ planes and the $\psi$ angles were than chosen according to this texture, to evaluate the residual stress. The residual stress found in the film was tensile, with a value of about $1600 \mathrm{MPa}$. The residual stress gradient in the Mo surface before and after Chromium deposition is given in Fig. 8. It can be seen that the mechanical polishing introduced a residual stress gradient in the Mo surface (before deposition). The data does not show that the high tensile residual stress found in the coating influences the relaxation of the compressive residual stress in the substrate (after deposition).

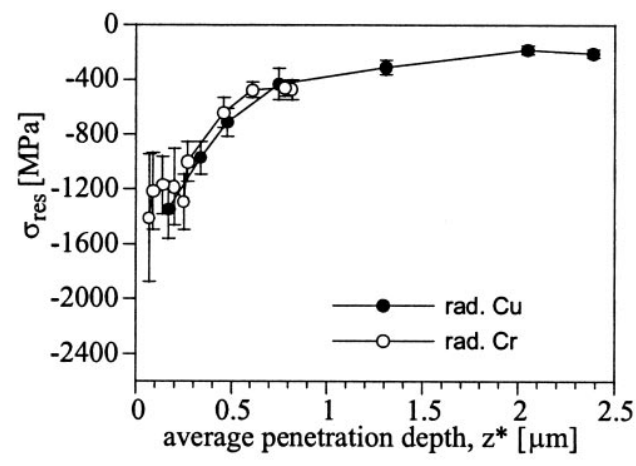

a) before chromium deposition

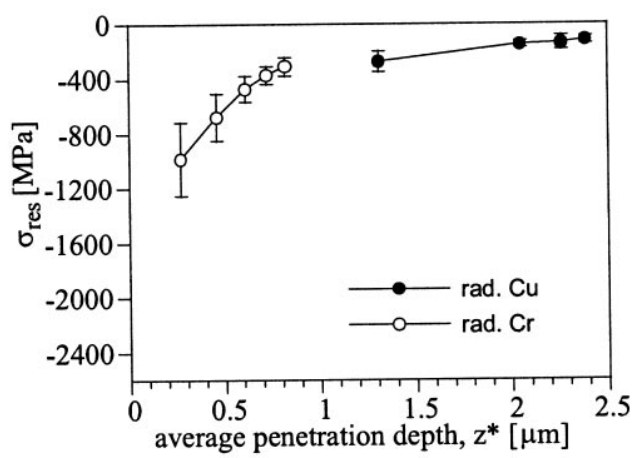

Fig. 8. Residual stress state in Mo polished surface: (a) before chromium deposition; (b) after chromium deposition. 
It can be seen that there are fewer points in Fig. 8(b) than in Fig. 8(a). Note that these acquisitions (Fig. 8b) were made after the film deposition, so it is clear that the peak intensity of the Mo substrate is weaker and decreases with the incident angle, and more information cannot be obtained. Fig. 8 also shows that the data obtained with both types of radiation is similar at the same penetration depth, which again confirms the precision of the PGIXRD.

\section{Conclusion}

The new geometry used in the PGIXRD is described in detail. The aim of this technique is to achieve a smaller X-ray penetration depth by decreasing the $\Omega$ angle, defined as the angle between the incident beam and the material surface. The evaluation of average residual stress corresponding to smaller depths, i.e. for each $\Omega$ angle, is then obtained by means of the conventional $\sin ^{2} \psi$ method using a $\Psi$-goniometer. This method imposes changes on the conventional equation used for the residual stress determination because of the asymmetric geometry. A modified equation is proposed in this paper.

The limitations of the technique were investigated for iron, nickel, molybdenum and copper powders using wavelengths produced by conventional X-ray tubes and by synchrotron radiation. It was shown that, for smaller incident angles, special care must be taken with regard to the peak treatment to apply the PGIXRD correctly. The peak shape changes for the lower angles and some peak treatment methods are more sensitive to such changes.

To minimise this problem, some experiments were performed using synchrotron radiation, because this beam is more coherent and has a higher intensity. However, the most important advantage of synchrotron radiation is the rapidity of acquisition and the symmetric shape of the resulting peak, thus avoiding the variation of the peak position at lower incident angles.

In this work the residual stress profile in a Mo substrate before and after the deposition of a PVD chromium coating was also determined, using $\mathrm{Cr} \mathrm{K} \alpha$ and $\mathrm{Cu} \mathrm{K} \alpha \mathrm{X}$-ray radiation, which permitted to analyse the surface layer at depths of between 2400 and $400 \mathrm{~nm}$. The experimental data lead to the following conclusions:

- A residual stress gradient was found in the surface of the Mo substrate, with a maximum average value of $-1600 \mathrm{MPa}$ at $400 \mathrm{~nm}$ and a minimum of $-200 \mathrm{MPa}$ in the bulk, induced by mechanical polishing.

- After chromium deposition, the residual stress state in the Mo substrate did not change. It seems that the high tensile stress found in the $\mathrm{Cr}$ film (about
$1600 \mathrm{MPa})$ did not have a significant influence on the relaxation of the residual stress in the Mo surface.

- The use of two different X-ray of wavelength confirms that the PGIXRD is a suitable technique for residual stress evaluation, as the same results were obtained with both wavelength.

\section{Acknowledgements}

M.J. Marques thanks the PRAXIS XXI Program (Portugal). Special thanks are due to $M$. Béssière and S. Lefebvre for their scientific support during LURE experiments.

\section{References}

[1] J. Lu, (ed.), Handbook of Measurement of Residual Stresses, Fairmont, Lilburn, 1996, pp. 71-131.

[2] C.I. Noyan, J.B. Cohen, Residual Stress, Measurement by Diffraction and Interpretation, Springer-Verlag, New York, 1987.

[3] V. Hauk, Structural and Residual Stress Analysis by Nondestructive Methods, Elsevier, Amsterdam, 1997.

[4] P. Predecki, X. Zhu, B. Ballard, Adv. X-Ray Anal. 36 (1993) 237-245.

[5] H. Ruppersberg, I. Detemple, J. Krier, Phys. Status Solidi A 116 (1989) 681-687.

[6] H. Ruppersberg, I. Detemple, J. Krier, Z. Krist. 195 (1991) 189-203.

[7] C. Genzel, Phys. Status Solidi A 146 (1994) 629-637.

[8] C. Genzel, W. Reimers, Phys. Status Solidi A 166 (1998) 751762.

[9] C. Genzel, Phys. Status Solidi A 165 (1998) 347-360.

[10] B. Eigenmann, B. Scholtes, E. Macherauch, Proceedings of the Third International Conference on Residual Stresses, ICRS3, Tokushima, Japan, July 23-26, 1991, Elsevier, London, 1992, pp. 601-606.

[11] B. Eigenmann, E. Macherauch, Nucl. Instr. Meth. Phys. Res. B 97 (1995) 92-97.

[12] C. Genzel, Proceedings of the Fifth International Conference on Residual Stress, ICRS-5, Linköping, Sweden, June 16-18, 1997, Institute of Technology, Linköping Universitet, 1997, pp. 514521.

[13] B. Ballard, P. Predecki, D. Braski, Adv. X-ray Anal. 37 (1994) 189-196.

[14] X. Zhu, P. Predecki, Adv. X-Ray Anal. 37 (1994) 197-203.

[15] T. Dümmer, B. Eigenmann, D. Löhe, Proceedings of the Third International Conference on Computer Methods and Experimental Measurements for Surface Treatment Effects; Surface Treatment, Oxford, UK, July 15-17, Computational Mechanics Publications, Southampton, Boston, 1997, 1997, pp. 371-379.

[16] A. Schubert, B. Kämpfe, E. Auerswald, B. Michel, in: V. Hauk, H.P. Hougardy, E. Macherauch, H.D. Tietz (Eds.), Residual Stresses, DGM Informationsgesellschaft mbH, Verlag, Oberusel, 1992, pp. 663-671.

[17] S. Bein, C.L. Calvez, J.L. Lebrun, Z. Metallkd. 89 (1998) 289-296.

[18] P. Nozières, D.E. Wolf, Z. Phys. B Cond. Matter 70 (1988) 399-407. 
[19] J.W. Gibbs, The Collected Works of J.W. Gibbs, Longmans, Green and Co., London, 1928.

[20] F.H. Streitz, R.C. Cammarata, K. Sieradzki, Phys. Rev. B 49 (1994) $10699-10706$.

[21] J.C. Woicik, J.G. Pellegrino, S.H. Southworth, P.S. Shaw, B.A. Karlin, C.E. Bouldin, K.E. Miyano, Phys. Rev. B 52 (1995) $2281-2284$.

[22] O. Brandt, K. Ploog, R. Bierwolf, M. Hohenstein, Phys. Rev. Lett. 68 (1992) 1339-1342.

[23] B. Ballard, X. Zhu, P. Predecki, D. Braski, Proceedings of the Fourth International Conference on Residual Stresses (ICRS-4), Baltimore, Maryland, USA, June 8-10, 1994, Society for Experimental Mechanics, Bethel, 1994, pp. 1133-1143.

[24] C. Houpert, H. Michaud, O. Valfort, Proceedings of the Fourth International Conference on Residual Stresses, ICRS-4, Balti- more, Maryland USA, June 8-10, 1994, Society for Experimental Mechanics, Bethel, 1994, pp. 245-251.

[25] H. Ruppersberg, Adv. X-ray Anal. 37 (1994) 235-244.

[26] C. Genzel, J. Appl. Cryst. 32 (1999) 770-778.

[27] C. Genzel, M. Broda, D. Dantz, W. Reimers, J. Appl. Cryst. 32 (1999) $779-787$.

[28] M. Barral, J.M. Sprauel, J.L. Lebrun, G. Maeder, S. Megtert, Adv. X-ray Anal. 27 (1983) 149-158.

[29] V. Hauk, E. Macherauch, Adv. X-ray Anal. 27 (1984) 81-99.

[30] V. Hauk, W.K. Krug, R.W.M. Oudeikoven, L. Pintschovius, Z. Metallkd. 79 (1988) 159-163.

[31] M. Zaouali, J.L. Lebrun, P. Gergaud, Surf. Coat. Tech. 50 (1991) 5-10.

[32] J. Pina, A. Dias, M. François, J.L. Lebrun, Surf. Coat. Technol. 96 (1997) 148-162. 